\title{
FOUR ASPECTS OF RELEVANCE IN SHARING LOCATION- BASED MEDIA: CONTENT, TIME, LOCATION AND NETWORK
}

\author{
Pasi Fränti, Jinhua Chen and Andrei Tabarcea \\ Speech \& Image Processing Unit, School of Computing, University of Eastern Finland, Joensuu \\ franti, jinchen, tabarcea@cs.joensuu.fi
}

Keywords: Data sharing, relevance, location-based applications.

Abstract: Sharing information via internet is popular but the key problem is how to find relevant information. Two new features are becoming more popular: location and the social network of the user. We hypothesize that the relevance of data is defined by four aspects: content, time, location, and user network. We study how the location aspect is used in a media-sharing service called MOPSI.

\section{INTRODUCTION}

Location-based services are becoming widely used due to the fast development of positioning systems in multimedia phones. Location provides additional information that can be expressed as a point of interest, route, or geographic area. The location can be considered information as itself but it is often attached to other data, and shared via location-based service or photo sharing site.

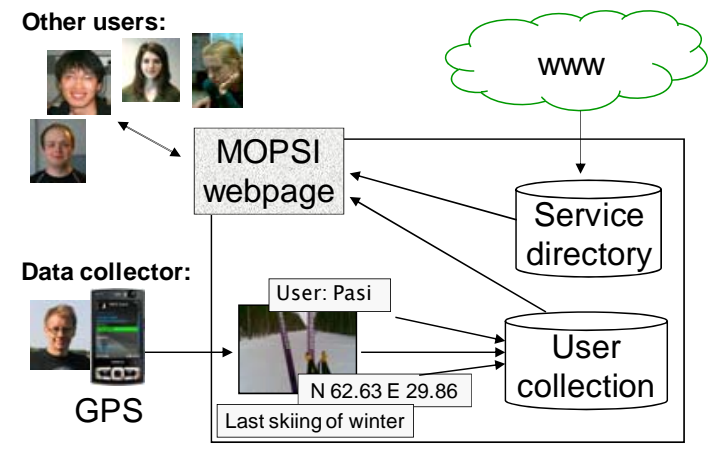

Figure 1: Diagram of the MOPSI data collection and services. Available on-line: http://cs.joensuu.fi/mopsi/

In this paper, we study mobile location-based media sharing via internet by a case study based on MOPSI service, which is prototype service for sharing location-based media. The overall structure of the system is outlined in Fig. 1 consisting of two main parts: user collection and service directory.

The main limitation of this kind of ad hoc information sharing is unawareness of the material of others especially if the users are not directly linked with each other. The data itself may be available in the service but the problem is how to find relevant data from service with a large number of users. We argue that relevance can be defined by the following aspects:

(1) Content of the data

(2) Location

(3) Time

(4) Author and his/her network

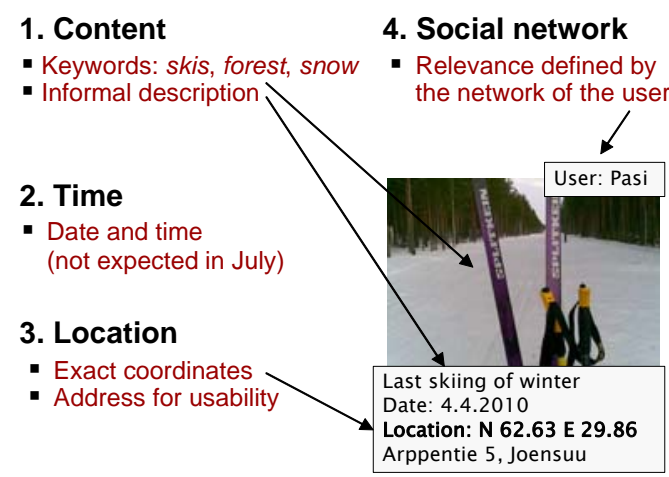

Figure 2: Four aspects of relevance in practice.

These four aspects are demonstrated in Fig. 2 by a concrete example where a person wanted to capture the following scenario. From the photo and its description we can see skis, forest and snow, which relate to wintertime activity. The data also reveals when and where the picture was taken. In $4^{\text {th }}$ April 2010, there was skiing tracks available, which was not self-evident even for citizens of Joensuu. Knowing a proper location was essential. The last piece of information is the identity of the user 
himself. Strangers may not benefit much of this information but those who know him and share the same hobbies are more likely to find this useful.

\section{CONTENT, TIME AND LOCATION}

We discuss the three main aspects of MOPSI system, of which location is an essential part. We describe the system as its current state, and discuss its design alternatives.

\subsection{Content}

Traditionally the relevance is defined by the content either by user-given keywords, or using a predefined format in database system, and then retrieved using SQL queries. This requires welldesigned static database where the service provider models the user behavior beforehand and provides information in form of service directory.

In internet, well-defined attributes are not used but relevant content can still be found from free text using search engine if the content matches to the keywords provided by the user. Tagging of the photos can also be done afterwards, but usually freeform textual explanation is simpler. It also serves the purpose of social media.

In MOPSI, free-form text description is supported instead of manual tagging. For browsing the data on web, queries based on time, location and content have been implemented. A simple recommendation framework is also in MOPSI based on user location and rating of the photos.

Further analysis of the relevance, content-based image retrieval could be done based on color, texture and shape features. Automatic image categorization, aims at converting visual content into a set of keywords to describe the content. In (Choudhury et al., 2009, Yu et al., 2009) both visual content and user tagging are jointly applied to recommend the group, where a photo should best fit in.

\subsection{Location}

Exploiting the location of the user has become popular due to wide availability of GPS positioning in multimedia phones. In case of lacking GPS, positioning can also be provided by cellular network of mobile phone, or even using the IP address for a rough estimation of location. Once the location is known, it gives significant additional relevance that can be utilized in several different ways. In MOPSI, location is the key element and it provides additional relevance in the following ways:

(1) Browsing data collection on a map

(2) Show location of other users

(3) Track the movements of the user

(4) Filter relevant search results for the service directory

Fig. 3 demonstrates the map view in MOPSI where photos have been clustered and then shown using GoogleMaps API.

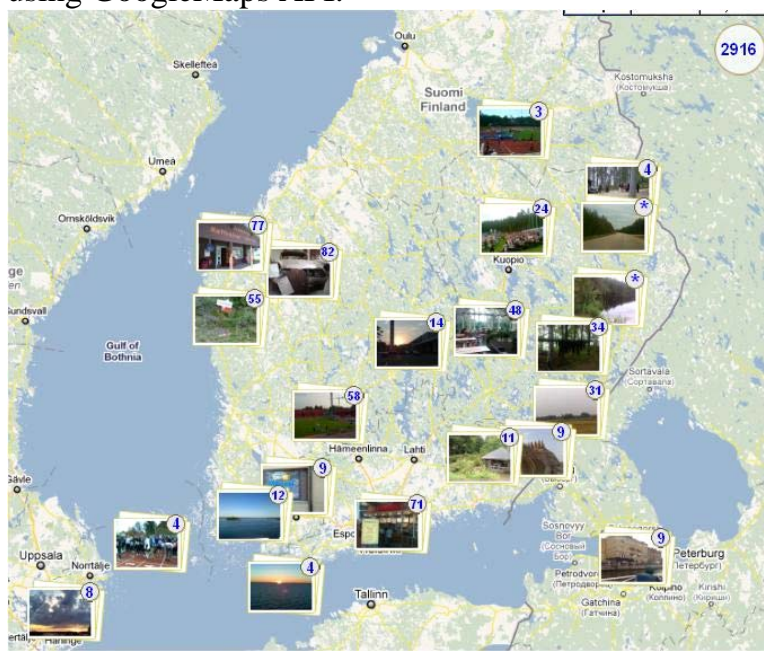

Figure 3: Map view of the data collection.

Location of users has been visualized in Fig. 4 using a so-called smart swap algorithm (Chen et al., 2010) that provides accurate clustering in real-time. For representing the clusters, approaches using icons, grids, Voronoi diagrams, and coloring by the density have been considered in (Delort, 2010). We use a color bubble attached with the text representing the most recent users in the cluster. The browsing is supported by zooming operation to get inside bigger clusters. Details of this solution will be reported later elsewhere.

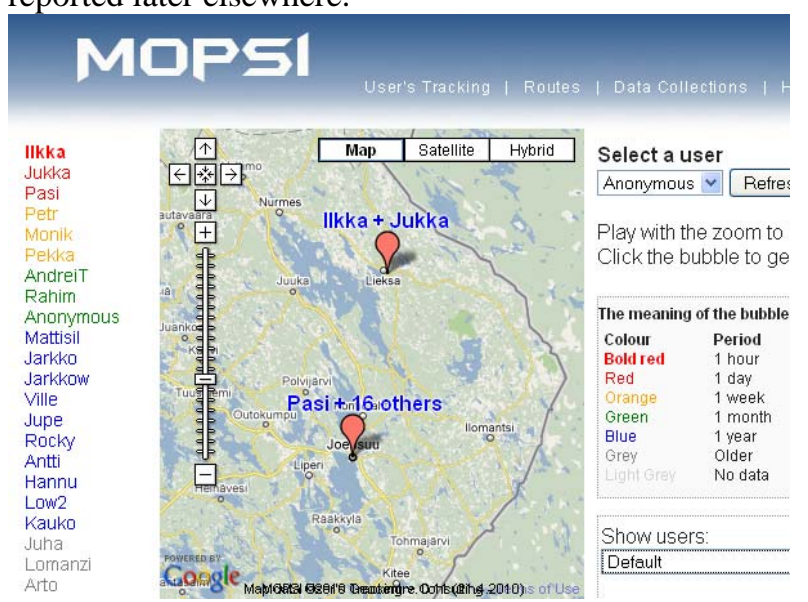

Figure 4: Map view of user locations. 
The collection can also be used as a part of service directory in MOPSI either in mobile phone or on web, see Fig. 5. Given location, user makes query by keyword, but instead of providing relevant search results by the content alone, results nearby are given if they exist in a local database (green), or found in the user collection (yellow).

Additional information (red) is provided by location-based search (Fränti et al., 2010), which is a combination of traditional location-based service and search engine. Following the idea in (Huitema and Fizzano, 2010), MOPSI allows user to transfer search engine results (red) into the service directory (green) by adding proper keywords similarly, and by using photos from the user collection (yellow).

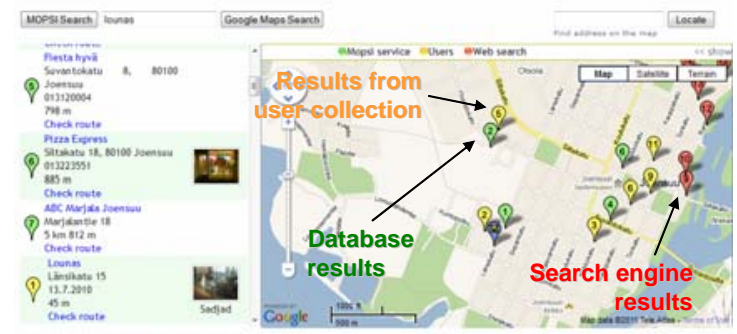

Figure 5: Web page interface to the service directory

\subsection{Time}

Time can be added to the relevance of the data in several ways. Firstly, the information may be relevant only within certain time period. A concert or a sport event happens in certain time and day, and it is essential information for participants. In photo collection, the information can also be relevant to know when the photo was taken. In MOPSI collection, we utilize this by providing time line view to the data as shown in Fig. 6. Similar layout was considered in (Setlur et al., 2009), with the addition that also links to Wikipedia are supported to provide more information besides just the photos.

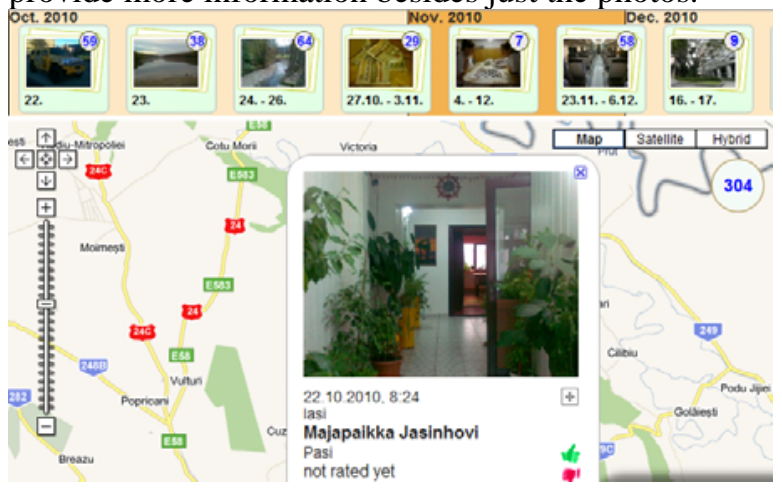

Figure 6: Time-line view of the data collection.
Secondly, the time and location themselves can be the essential data from an exercise session. For example, the jogging track shown in Fig. 7 records the length, duration and average speed. This is typical book-keeping for a long distance runner in his training. Although specialized GPS sport trackers exist, the use of MOPSI service and mobile phone allows automatic sending of the data into the server for user convenience. Moreover, photos can also be taken from the same session by the same device, and presented later jointly with the trajectory of the user as proposed in (Petit et al., 2008).

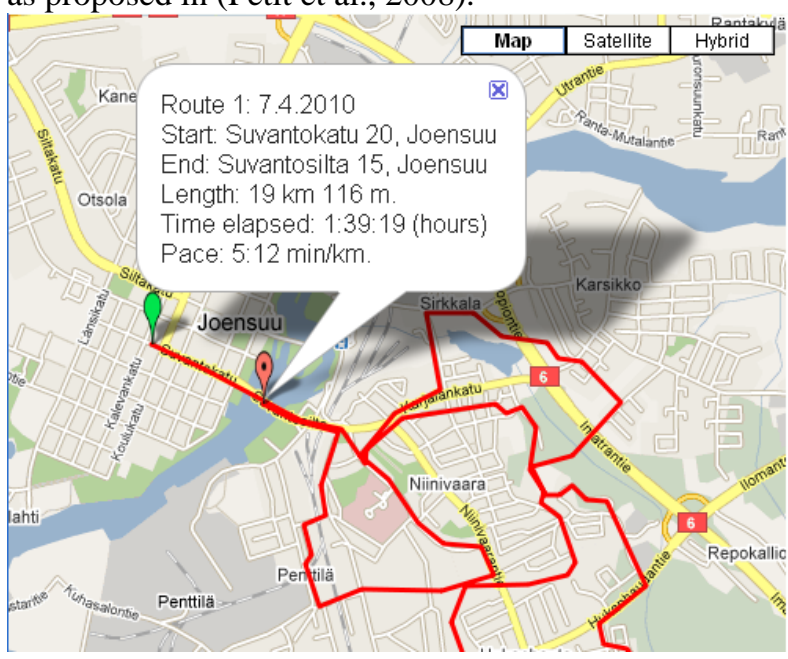

Figure 7: Joint time and location for tracking sport activity.

In MOPSI collection, tracking user's routes is one of the main functions. The web interface provides also navigation from the current location to the location of the search result using GoogleMaps API based on road maps. An interesting idea for future consideration would be to use the route collection of all users to offer better navigation for pedestrians and hikers instead of the road network more suitable for cars (Kasemsuppakorn et al., 2009).

Third possibility to utilize the time information is to consider the age of data. The newer the information the more likely it is still valid as the life expectancy of cafeterias, for example, in typical metropolitan area are often measured in months rather than in years. Moreover, information such as weather condition is needed right here and right now, so to speak. In Fig. 1, the skiing condition is recorded for $4^{\text {th }}$ April, but it hardly relevant for users in July. 


\section{EXPERIMENTS}

We next give overview of the data in the user collection so far as on $25^{\text {th }}$ October 2010. The collection includes lots of test photos, and the number of users is small, which may somewhat skew the results. Nevertheless, some trends and observations can be seen.

In total, there were 3589 photos of which most are city views (839), then pictures of nature (801) and other people (279). Few pictures are also taken from events (90), documents (40) and animals (59). In addition, there are photos that are counted as test photos or failure pictures.

Another point of view is what kind of descriptions has been typed in by the users. Due to the experimental stage, a large amount of the photos (27\%) are without any description. The lack of descriptions is also caused by the difficulty to type by mobile phone, but descriptions can be added later from the web interface.

Among the photos that have some kind of description, significant amount of photos (35\%) have just garbage, some test word (Symbian_test), or very generic object description (Mug, Wires, Mouse) indicating test use. In total, $65 \%$ of all photos have a meaningful description. Mostly documented descriptions are travel photos of places (685), nature (579), general objects (263), architecture (212) people (210), and few general descriptions of events and animals.

People are often described by their names, or by their roles (runner, floorball player). Only few are related to place (Untung / STMIK), age (Young Andrei) or relationship to the person (my son Amir).

Events are significantly more often found in the user description than could be concluded by content analysis alone. In our case, events include mostly work-related meetings described by their acronyms (ecse, abi, mopsi meeting, ubiikki) but also running competition (Aland half marathon) and actions attached with feelings (quality time in skiing elevator).

Another difference between content and user description are travel photos. The location is not easy to recognize from content but it could be concluded from the positioning data. For example, Clarke Quay, Geger beach, Suceava, Tahkovuori and Aholansaari are locations whereas the following descriptions include additional details: Petronas Towers (building complex), Heureka (science center), Singapore flier (Ferris wheel) and Olavin linna (castle). The extreme case is Musta Pekka mutkan takana (Black Pete behind the curve) where
Black Pete is the name of a particular slope in Tahko skiing resort.

Table 1: Distribution of keywords (tags) used in Picasa and Flickr, in comparison to the user descriptions of MOPSI collection.

\begin{tabular}{|c|c|c|c|c|}
\hline \multirow[t]{2}{*}{ Description: } & \multirow{2}{*}{ Picasa } & \multirow{2}{*}{ Flickr } & \multicolumn{2}{|c|}{ MOPSI } \\
\hline & & & All & Real \\
\hline Places & --- & $28 \%$ & $21 \%$ & $32 \%$ \\
\hline Events and action & $31 \%$ & $17 \%$ & $5 \%$ & $7 \%$ \\
\hline People & $6 \%$ & $7 \%$ & $6 \%$ & $10 \%$ \\
\hline Objects & --- & $5 \%$ & $8 \%$ & $12 \%$ \\
\hline $\begin{array}{l}\text { Architecture and } \\
\text { nature }\end{array}$ & $25 \%$ & $21 \%$ & $23 \%$ & $37 \%$ \\
\hline Animals & --- & $3 \%$ & $2 \%$ & $2 \%$ \\
\hline Other .... & $20 \%$ & $16 \%$ & --:- & $0 \%$ \\
\hline Garbage & $19 \%$ & $2 \%$ & $35 \%$ & -- \\
\hline
\end{tabular}

Table 1 compares the textual description used in MOPSI with two other photo sharing sites. The main difference is that, in MOPSI, location is provided automatically without any user interaction.

In Picasa, users provide the location by dragging the photo on GoogleMap. Keywords and location are thus provided explicitly as two different entities, and consequently, users tend not to type any location related keywords. Flickr has somewhat more complicated interface based on Yahoo! Maps. Only a predefined set of keywords are allowed, which explains the quality of tags (only $2 \%$ garbage).

Despite the automatic positioning in MOPSI, it does not reflect on the distribution of the type of descriptions written. Unlike in Picasa, users still tend to describe the location anyway for travel pictures, probably because the position is not confirmed in the device, but it happens hidden in the background. Overall, the distribution of topics is rather similar to that of Flickr. There are slightly more people and objects described, but these could be just artifacts from the system being at testing stage.

For photo collecting, two mobile applications were developed (Java and Symbian $\mathrm{C}++$ ). A large number of failures were caused by the Java version, which lacks several important features. Firstly, there is an unavoidable delay from the click sound and when the photo is actually taken. People tend to move the camera right after they hear the sound and before the actual picture will be taken. Secondly, Auto-focus supported by Symbian helps a lot with picture quality but it was not available in Java. Other typical failures originate from low quality cameras that do not work well in low illumination. Few damaged pictures were caused by irrecoverable transmission error. Samples are shown in Fig. 8. 

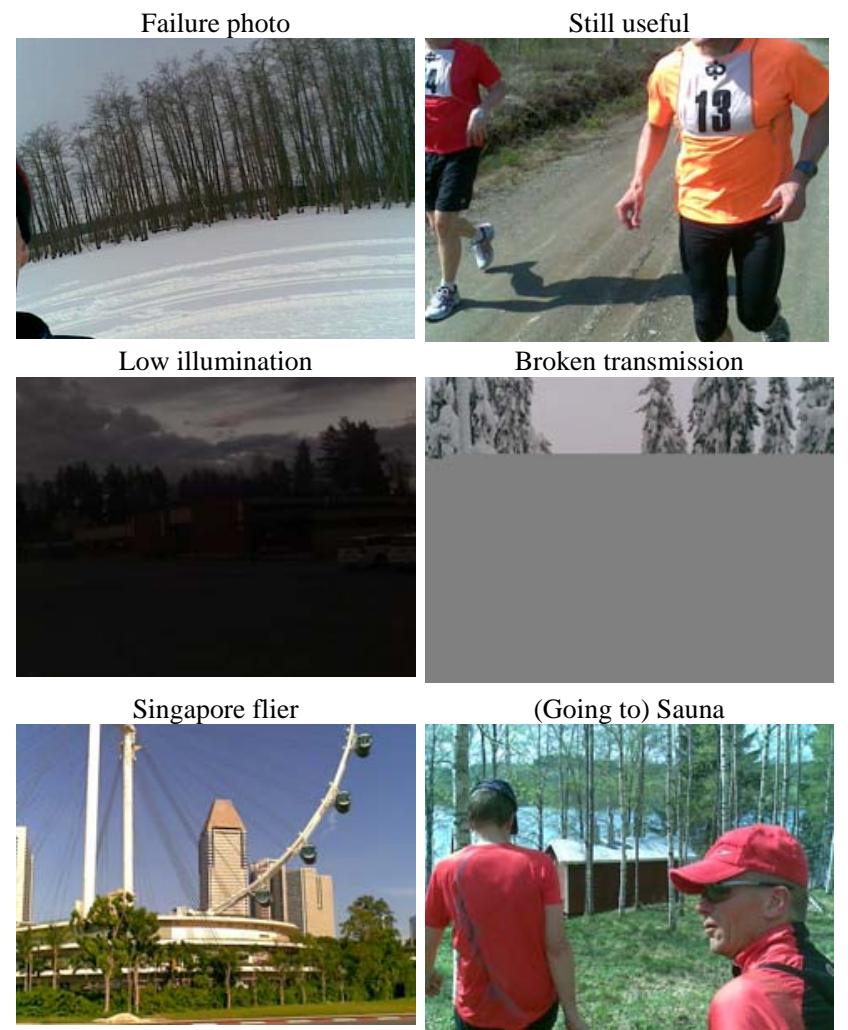

No keywords needed
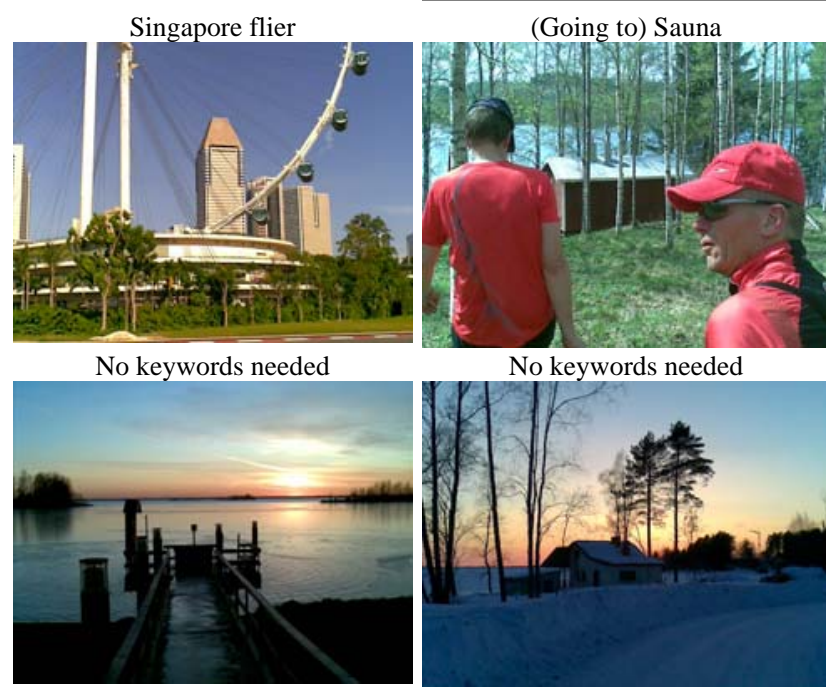

No keywords needed

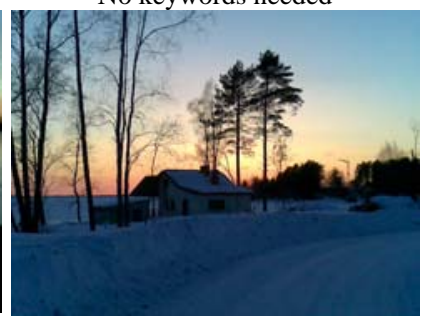

Figure 8: Photos of the first row are examples of software problems (click sound), the second row of low illumination and broken transmission problems. The rest are successful photos.

\section{CONCLUSIONS}

We have presented a case study of MOPSI locationbased media collection and sharing service, and studied how different aspects of relevance appear in the system. So far the system has been used for collecting user data (mainly photos and routes), served as a test bench of new ideas, and a prototype service directory. In all these, the location is a key factor.

The media collection tool is also in professional use by partnering companies for documenting purpose, and can be used later for mobile locationbased games and an educational tool for teaching principles of GIS, and other classes such as biology. The fourth aspect of relevance, social network, will be studied in future.

\section{REFERENCES}

Choudhury M.D., Sundaram H., Lin Y.-R., John A., Seligmann D.D., "Connecting content to community in social media via image content, user tags and user communication”, ICME 2009, 1238-1241, New York City, July 2009.

Yu J., Joshi D., Luo J., "Connecting people in photosharing sites by photo content and user annotations", ICME 2009, 1464-1467, New York City, July 2009.

Chen J., Zhao Q., and Fränti P., "Smart swap for more efficient clustering", Int. Conf. Green Circuits and Systems (ICGCS'10), Shanghai, China, June 2010.

Delort J.-Y., "Vizualizing large spatial datasets in interactive maps", IEEE Int. Conf. Advanced Geographic Information Systems, Applications, and Services, St. Maarten, Netherlands Antilles, 33-38, Feb 2010.

Fränti P., Tabarcea A., Kuittinen J., Hautamäki V., "Location-based search engine for multimedia phones", IEEE Int. Conf. on Multimedia \& Expo (ICME'10), Singapore, July 2010.

Huitema P. and Fizzano P., "A Crawler for Local Search”, IEEE Int. Conf. Digital Society (ICDS), St. Maarten, Netherlands Antilles, 86-91, Feb 2010.

Setlur V., Battestini A., Ding X., "Travel scrapbooks: creating rich visual travel narratives”, ICME 2009, 1314-1317, New York City, July 2009.

Petit M., Claramunt C., Ray C. and Calvary G., "A design process for the development of an interactive and adaptive GIS”, W2GIS, 96-106, Shanghai, China 2008.

Kasemsuppakorn P., Karimi H.A., "Pedestrian network data collection through location-based social networks", Collaborate COM, Crystal City, Washington DC, Nov 2009. 